\title{
不同模拟体液对硼硅酸盐生物活性玻璃 基骨水泥矿化性能的影响
}

\author{
林子扬 $^{1}$, 常宇辰 ${ }^{2}$, 吴章凡 ${ }^{1}$, 包 荣 $^{3}$, 林文庆 ${ }^{3}$, 王德平 ${ }^{1}$
}

(1. 同济大学 材料科学与工程学院, 上海 201804; 2. 伽蓝(集团)股份有限公司, 上海 200030; 3. 上海三悠树脂有 限公司，上海 201818)

摘 要: 硼硅酸盐生物活性玻璃基(Borosilicate bioactive glass-based, BBG)骨水泥由于其优异的生物活性和生物降解 性，在治疗骨质疏松性骨折以及骨肿瘤、骨创伤、骨髓炎等疾病方面具有重要的应用前景，受到人们的广泛关注。 为进一步了解氨基酸对其植入生物体内后的矿化影响, 本研究在常规的 SBF 溶液中添加了不同种类及浓度的氨基 酸物质, 重点研究对植入体表面形貌的影响。同时为在矿化过程中同步形成白磷钲矿(Whitlockite, WH)和羟基磷灰 石(hydroxyapatite, HA), 调整了 $\mathrm{SBF}$ 溶液的温度以及酸碱度和 $\mathrm{Mg}^{2+}$ 浓度, 研究了不同 $\mathrm{SBF}$ 溶液中 BBG 骨水泥表面 形成的矿化产物。研究结果表明, 不同的氨基酸及浓度的变化对矿化产物的影响有较大差异, 天冬氨酸和赖氨酸的 浓度变化影响矿物的长径比, 而甘氨酸对矿物形貌的影响较小。将嗍硅酸盐生物活性玻璃压片放置在 $70{ }^{\circ} \mathrm{C}$ 下的高 $\mathrm{Mg}^{2+}$ 浓度的酸性 $(\mathrm{pH}=3.5) \mathrm{SBF}$ 溶液中浸泡一定时间后, 能够获得 $\mathrm{HA} / \mathrm{WH}$ 的复相矿物。

关 键 词: 模拟体液; 氨基酸; 硼硅酸盐生物活性玻璃基骨水泥; 白磷钻矿; 羟基磷灰石

中图分类号: TQ171 文献标志码: A

\section{Effect of Different Simulated Body Fluid on Mineralization of Borosilicate Bioactive Glass-based Bone Cement}

\author{
LIN Ziyang ${ }^{1}$, CHANG Yuchen ${ }^{2}$, WU Zhangfan ${ }^{1}$, BAO Rong ${ }^{3}$, LIN Wenqing ${ }^{3}$, WANG Deping ${ }^{1}$ \\ (1. School of Materials Science and Engineering, Tongji University, Shanghai 201804, China; 2. JALA (Group) Co., Ltd, Shanghai \\ 200030, China; 3. Shanghai Sanyu Resin Co., Ltd, Shanghai 201818, China)
}

\begin{abstract}
Borosilicate bioactive glass-based (BBG) bone cement has important application prospects in the treatment of osteoporotic fractures, bone tumors, bone trauma, osteomyelitis, and other diseases due to its excellent bioactivity and biodegradability. To further understand the factors affecting its mineralization ability after implantation into human body, three kinds of amino acid were added into the conventional simulated body fluids (SBF) solution. Furthermore, to get whitlockite (WH) and hydroxyapatite (HA) simultaneously during the mineralization process, the environmental temperature, $\mathrm{pH}$ and $\mathrm{Mg}^{2+}$ concentration of $\mathrm{SBF}$ were adjusted. The mineralized products of BBG bone cement after being immersed in different SBF solutions were characterized. It is found that the effects of amino acids on mineralization products are quite different, of which aspartic acid and lysine affect the aspect ratio
\end{abstract}

收稿日期：2020-08-20；收到修改稿日期：2020-09-18; 网络出版日期：2020-10-10

基金项目：国家重点研发计划(2018YFC1106300); 上海三悠树脂有限公司(05002450376)

National Key Research and Development Project (2018YFC1106300); Shanghai Sanyu Resin Co., Ltd (05002450376)

作者简介：林子扬(1996-), 男, 硕士研究生. E-mail: lzyyymx@163.com LIN Ziyang(1996-), male, Master candidate. E-mail: lzyyymx@163.com

通信作者：王德平，教授.E-mail: wdpshk@tongji.edu.cn WANG Deping, professor. E-mail: wdpshk@tongji.edu.cn 
of the mineral, but glycine does not affect the morphology of the mineral. After the borosilicate bioactive glass tablet being immersed in an acidic (pH 3.5) SBF solution with a high $\mathrm{Mg}^{2+}$ content at $70{ }^{\circ} \mathrm{C}$ for a certain period, HA/WH complex mineral can be obtained.

Key words: simulated boby fluid; amino acid; borosilicate bioactive glass-based bone cement; whitlickite;

hydroxyapatite

生物材料浸泡在模拟体液(Simulated body fluid, $\mathrm{SBF}$ )中能否形成着基磷灰石(Hydroxyapatite, HA)是 预测其生物活性的重要依据之一, 也是判断其植入 体内后能否与骨组织发生化学键合的重要条件之 - ${ }^{[1]}$ 。SBF 具有与血浆近乎相同的离子浓度, 但又 有些微不同的成分。血浆中除了无机盐外, 还存在 氨基酸、葡萄糖以及蛋白质等有机分子 ${ }^{[2]}$ 。Palazzo 等 ${ }^{[3]}$ 研究了丙氨酸、精氨酸、天冬氨酸三种氨基酸 与仿生缺钙 $\mathrm{HA}$ 生长的协同作用, 发现非极性氨基 酸会影响 HA 表面结构的有序性, 精氨酸或天冬氨 酸则主要使得缺钙 HA 呈针状, 表面变得更加粗糙。 Huang 等 ${ }^{[4]}$ 研究了天冬氨酸对 HA 生长的抑制机理, 发现天冬氨酸被吸附在 HA 的特定表面, 与表面的 $\mathrm{Ca}^{2+}$ 离子相互作用, 从而阻碍生长位点和抑制 $\mathrm{HA}$ 晶体的生长。Martins 等 ${ }^{[5]}$ 的研究则发现, 葡萄糖是 HA 纳米结构降解过程中的活性化学因子。而釉原 蛋白衍生肽的存在会抑制溶液中无机离子的成核, 且抑制作用随着分子尺寸的增大而增强 ${ }^{[6]}$ 。这些研 究都很好地证明了浸泡液中的有机分子对 HA 矿物 的形貌及生长速率具有调控作用。然而, 对类似喼 硅酸盐生物玻璃等多组分非晶态材料在含有蛋白质 等物质的模拟体液中的矿化行为尚未见报道。嗍硅 酸盐生物活性玻璃(Borosilicate Bioglass, BBG)骨水 泥在植入体内后能够快速原位固化, 为骨缺损部位 提供力学支撑, 同时还具有良好的生物活性和操作 性能, 在体液环境下矿化形成 HA, 促进骨组织的 修复与再生 ${ }^{[7-9]}$ 。因此, 作为一类新型的可注射骨修 复材料, 研究其在不同 SBF 溶液中浸泡后的矿化性 能及其影响因素对于进一步理解生物玻璃材料与生 物功能的关联性, 掌握其植入体内后参与骨修复的 精细过程具有重要意义。

此外, 在人体的骨组织中, 除了存在大量的 HA 矿物之外, 白磷钙矿 (Whitlockite, WH) 也是骨和牙 齿中含量次多的无机矿物, 占骨骼的 $20 \%$ 和牙本质 的 26\% 28\%, 在青少年和成骨的早期阶段, WH 占 的比例更高, 在骨组织的生长过程中起着重要的促 进作用 ${ }^{[10-12]}$ 。Wang 等 ${ }^{[13]}$ 制备了含有 WH 的 BBG 骨 水泥, 研究了 WH 矿物对骨水泥生物性能的影响。他
们发现添加了 WH 的玻璃基骨水泥在大鼠骨髓间充 质干细胞(rBMSCs)共培养中表现出良好的促细胞 增殖和成骨分化能力。但是, 由于玻璃基骨水泥中 的 HA 物相是在 SBF 中通过降解一沉淀的矿化过程 中形成的, 与添加的 $\mathrm{WH}$ 矿物相比较, 无论在生 物活性方面, 还是在材料的物理化学性能方面都会 有一定的差异。若能够模拟自然骨组织中的 HA 与 WH 两相矿物共存的结构, 即如果能够让 BBG 骨水 泥浸泡在 SBF 中同时矿化形成 HA 和 WH, 将有利 于进一步了解材料与组织微环境间的宿主反应机理, 改善骨水泥的生物性能及骨组织修复能力。

据此, 本研究通过在传统 SBF 溶液中添加氨基 酸的方式研究不同种类及浓度的氨基酸对 BBG 骨 水泥矿化过程及产物的影响, 在此基础上, 为在矿 化过程中同步获得 HA 和 $\mathrm{WH}$ 复相矿化产物, 调整 $\mathrm{SBF}$ 溶液的离子浓度及 $\mathrm{pH}$ 和温度等工艺条件, 对 浸泡后的 BBG 表面产生的物相进行表征分析。

\section{1 实验方法}

\section{1 实验试剂}

碳酸钠 $\left(\mathrm{Na}_{2} \mathrm{CO}_{3}\right)$ 、碳酸钾 $\left(\mathrm{K}_{2} \mathrm{CO}_{3}\right)$ 、碳酸钻 $\left(\mathrm{CaCO}_{3}\right)$ 、碱式碳酸镁 $\left(\mathrm{Mg}(\mathrm{OH})_{2} \cdot \mathrm{MgCO}_{3}\right)$ 、嗍酸 $\left(\mathrm{H}_{3} \mathrm{BO}_{3}\right)$ 、二氧化硅 $\left(\mathrm{SiO}_{2}\right)$ 、磷酸二氢钠 $\left(\mathrm{NaH}_{2} \mathrm{PO}_{4}\right)$ 、 磷酸氢二钠 $\left(\mathrm{Na}_{2} \mathrm{HPO}_{4}\right)$ 、甘氨酸 $\left(\mathrm{C}_{2} \mathrm{H}_{5} \mathrm{NO}_{2}\right)$ 、海藻酸 钠 $\left(\left(\mathrm{C}_{6} \mathrm{H}_{7} \mathrm{NaO}_{6}\right)_{n}\right)$ 均为化学纯, $L$-天冬氨酸 $\left(\mathrm{C}_{4} \mathrm{H}_{7} \mathrm{NO}_{4}\right)$ 和 $L$-赖氨酸 $\left(\mathrm{C}_{6} \mathrm{H}_{14} \mathrm{~N}_{2} \mathrm{O}_{2}\right)$ 为生物试剂, 均购自中国医 药上海化学试剂公司。

\subsection{BBG 的制备}

采用熔融 - 冷却 法 ${ }^{[14]}$ 制备了组成为 $6 \mathrm{Na}_{2} \mathrm{O} \cdot 8 \mathrm{~K}_{2} \mathrm{O} \cdot 8 \mathrm{MgO} \cdot 22 \mathrm{CaO} \cdot 36 \mathrm{~B}_{2} \mathrm{O}_{3} \cdot 2 \mathrm{P}_{2} \mathrm{O}_{5} \cdot 18 \mathrm{SiO}_{2}$ 的 $\mathrm{BBG}$ 。将各组分对应的氧化物、氯化物、碳酸盐、 磷酸盐、硫酸盐等原料均匀混合后, 置于铂金坩埚 中, 在 $1200{ }^{\circ} \mathrm{C}$ 的高温炉中熔制 $1 \mathrm{~h}$ 。将熔融的玻璃 淬火后得到玻璃块体, 经破碎后分散在无水乙醇中 球磨 $24 \mathrm{~h}$, 并在 $60{ }^{\circ} \mathrm{C}$ 恒温鼓风干燥箱中干燥, 最终 获得嗍硅酸盐生物玻璃粉末样品, 作为骨水泥的 固相。 


\section{$1.3 \mathrm{BBG}$ 骨水泥的制备}

将重量百分比为 $2 \%$ 海藻酸钠、 $1 \%$ 葡萄糖酸内 酯、3\%磷酸氢二钠溶解在去离子水中, 充分混合均 匀后得到海藻酸钠溶液, 作为骨水泥的液相 ${ }^{[8]}$, 利 用海藻酸钠与 $\mathrm{Ca}^{2+}$ 的胶凝作用增强骨水泥力学强 度。嗍硅酸盐生物玻璃粉末与海藻酸钠溶液按照固 液比 $2.5 \mathrm{~g} / \mathrm{mL}$ 混合搅拌约 $60 \mathrm{~s}$ 后, 将骨水泥浆料填 充到聚四氟乙烯圆柱形模具 $(\phi 6 \mathrm{~mm} \times 12 \mathrm{~mm})$ 中成型, 在温度 $37{ }^{\circ} \mathrm{C}$ 、相对湿度 $100 \%$ 的恒温恒湿箱中养护 固化 $24 \mathrm{~h}$, 得到 BBG 骨水泥样品。

\section{4 含氨基酸 SBF 溶液的制备及 BBG 骨水泥 的浸泡和表征}

为了更有效地研究氨基酸对 BBG 骨水泥矿化 过程的影响, 调整浸泡液的离子浓度为 1.5 倍标准 SBF 的离子浓度, 制备成 1.5 倍的 SBF(1.5SBF), SBF 组成如表 1 所示(按 $1000 \mathrm{~mL}$ 计 $)^{[1,15]}$ 。分别将天冬氨 酸、甘氨酸、赖氨酸溶解在 $1.5 \mathrm{SBF}$ 中, 配置成 0.1 和 $0.2 \mathrm{~mol} / \mathrm{L}$ 的溶液, 用 $\mathrm{HCl}$ 或 Tris 调整溶液的 $\mathrm{pH}$ 至 7.4。BBG 骨水泥按照固液比 $0.02 \mathrm{~g}: 1 \mathrm{~mL}$ 的比 例浸泡在含有氨基酸的 $1.5 \mathrm{SBF}$ 中, 放入 $37{ }^{\circ} \mathrm{C}$ 恒温 恒湿箱中培养, 每隔 $48 \mathrm{~h}$ 更换全部溶液。对照组浸 泡溶液为不加氨基酸的 $1.5 \mathrm{SBF}$ 。记录浸泡时间为 2 、 6、10、 $14 \mathrm{~d}$ 时不同实验组溶液的 $\mathrm{pH}$ 变化。将浸泡 不同时间后的骨水泥样品取出, 用无水乙醇和去离 子水清洗、干燥, 之后在样品表面喷金, 采用场发射 扫描电子显微镜(SEM, Quanta 200 FEG)观察浸泡后 的骨水泥样品表面形貌, 并通过 X 射线衍射 XRD, SmartLab 9)对骨水泥表面浸泡产物的物相进行检测, 扫描速率 $6\left(^{\circ}\right) / \mathrm{min}$ 、扫描范围 $2 \theta=10^{\circ} \sim 80^{\circ}$ 。

\subsection{BBG 的复相矿化实验}

为获取 $\mathrm{HA} / \mathrm{WH}$ 的复相矿化产物, 实验调整了 浸泡 SBF 的成分、 $\mathrm{pH}$ 和温度。考虑到 $\mathrm{HA}$ 在 $\mathrm{pH}>$ 4.2 的环境中形成最稳定的磷酸钙矿物, 而白磷钙 矿在 $\mathrm{pH}<4.2$ 时才有可能形成, 因此将浸泡溶液的 $\mathrm{pH}$ 调整至 3.5 左右 ${ }^{[16]}$ 。在 $\mathrm{pH} 3.5$ 的酸性条件下, 嗍 硅酸盐生物玻璃降解迅速会导致浸泡溶液 $\mathrm{pH}$ 迅速
升高, $1 \mathrm{~h}$ 时内 $\mathrm{pH}$ 可以从 3.5 升高至 6 以上，从而影 响浸泡液的酸碱稳定性。因此本研究采用了自行设 计的动态浸泡装置进行矿化实验。此外, 海藻酸钠 中的 $\mathrm{G}$ 嵌段会与 $\mathrm{Ca}^{2+} 、 \mathrm{Mg}^{2+}$ 等二价阳离子发生离子 交换而胶凝 ${ }^{[17]}$, 为了避免白磷钙矿晶体生长过程受 到海藻酸钠的影响, 研究直接将 BBG 压片作为矿 化实验的浸泡物。同时为了达到白磷钙矿结晶的过 饱和度与其沉积的温度要求 ${ }^{[18]}$, 如表 1 中的 2Mg-1.5SBF 所示, 调整了浸泡溶液中的 $\mathrm{Mg}^{2+}$ 浓度, 并将矿化反应的实验装置放置在 $70{ }^{\circ} \mathrm{C}$ 水浴锅中, 分别进行了 7 和 $10 \mathrm{~d}$ 的动态浸泡。用 FESEM (Quanta 200 F) 观察矿化产物的形貌, 用 XRD (SmartLab 9)和 TEM(H-800)表征产物的物相和晶体 结构。

\section{2 结果与讨论}

\section{1 不同氨基酸对浸泡物 BBG 骨水泥表面形 貌的影响}

为了模拟生物体微环境中的氨基酸对 BBG 骨 水泥矿化的影响, 选择了三种氨基酸, 分别是天冬 氨酸(酸性氨基酸)、甘氨酸(中性氨基酸)、赖氨酸(碱 性氨基酸)。图 1(a)显示浸泡前骨水泥的表面形貌, 表明海藻酸钠与嗍硅酸盐玻璃混合后了结合良好。 图 1(b)显示浸泡前骨水泥的 XRD 图谱呈现明显的 非晶态物质的馒头峰形态。将骨水泥样品分别浸泡 在含有 $0.1 \mathrm{~mol} / \mathrm{L}$ 不同氨基酸的 $1.5 \mathrm{SBF}$ 中 (对照组为 不含氨基酸的 1.5SBF)一定时间后，表面形貌(图 2) 显示, 浸泡 $5 \mathrm{~d}$ 的骨水泥表面已经形成了颗粒状的 矿物, 其中天冬氨酸组和赖氨酸组中的样品表面分 别形成了条状和短棒状的颗粒, 而甘氨酸组和对照 组样品表面是偏球形的颗粒。但随着浸泡时间的延 长, 浸泡 $7 \mathrm{~d}$ 的各组样品表面均生长了均匀的矿物; 浸泡 $14 \mathrm{~d}$, 天冬氨酸组样品表面形成了由条状矿物 组成的片层状结构, 甘氨酸组样品表面形成了颗粒 较细小的网状结构, 赖氨酸组样品则形成了由尺寸

表 1 不同 SBF 溶液的组成 $/\left(\mathbf{m m o l} \cdot \mathrm{L}^{-1}\right)$

Table 1 Composition of different SBFs $/\left(\mathbf{m m o l} \cdot \mathrm{L}^{-1}\right)$

\begin{tabular}{ccccccccccc}
\hline Ion & $\mathrm{Na}^{+}$ & $\mathrm{K}^{+}$ & $\mathrm{Mg}^{2+}$ & $\mathrm{Ca}^{2+}$ & $\mathrm{Cl}^{-}$ & $\mathrm{HCO}_{3}^{-}$ & $\mathrm{HPO}_{4}^{2-}$ & $\mathrm{SO}_{4}^{2-}$ & $\mathrm{pH}^{2-}$ \\
\hline Blood plasma & 142.0 & 5.0 & 1.5 & 2.5 & 103.0 & 27.0 & 1.0 & 0.5 & $7.2-7.4$ \\
SBF & 142.0 & 5.0 & 1.5 & 2.5 & 147.8 & 4.2 & 1.0 & 0.5 & 7.4 \\
1.5SBF & 213.0 & 7.5 & 2.25 & 3.75 & 221.7 & 6.3 & 1.5 & 0.75 & 7.4 \\
2Mg-1.5SBF & 213.0 & 7.5 & 4.5 & 3.75 & 226.2 & 6.3 & 1.5 & 0.75 & 3.5 \\
\hline
\end{tabular}



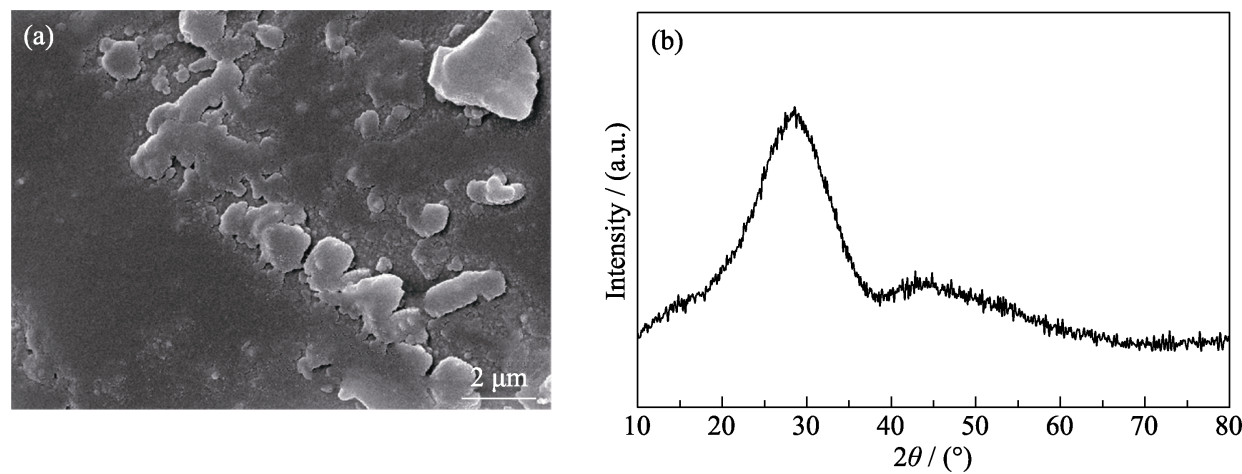

图 1 浸泡前骨水泥的(a)表面形貌及(b )XRD 图谱

Fig. 1 (a) Surface morphology and (b) XRD pattern of BBG bone cement before being soaked
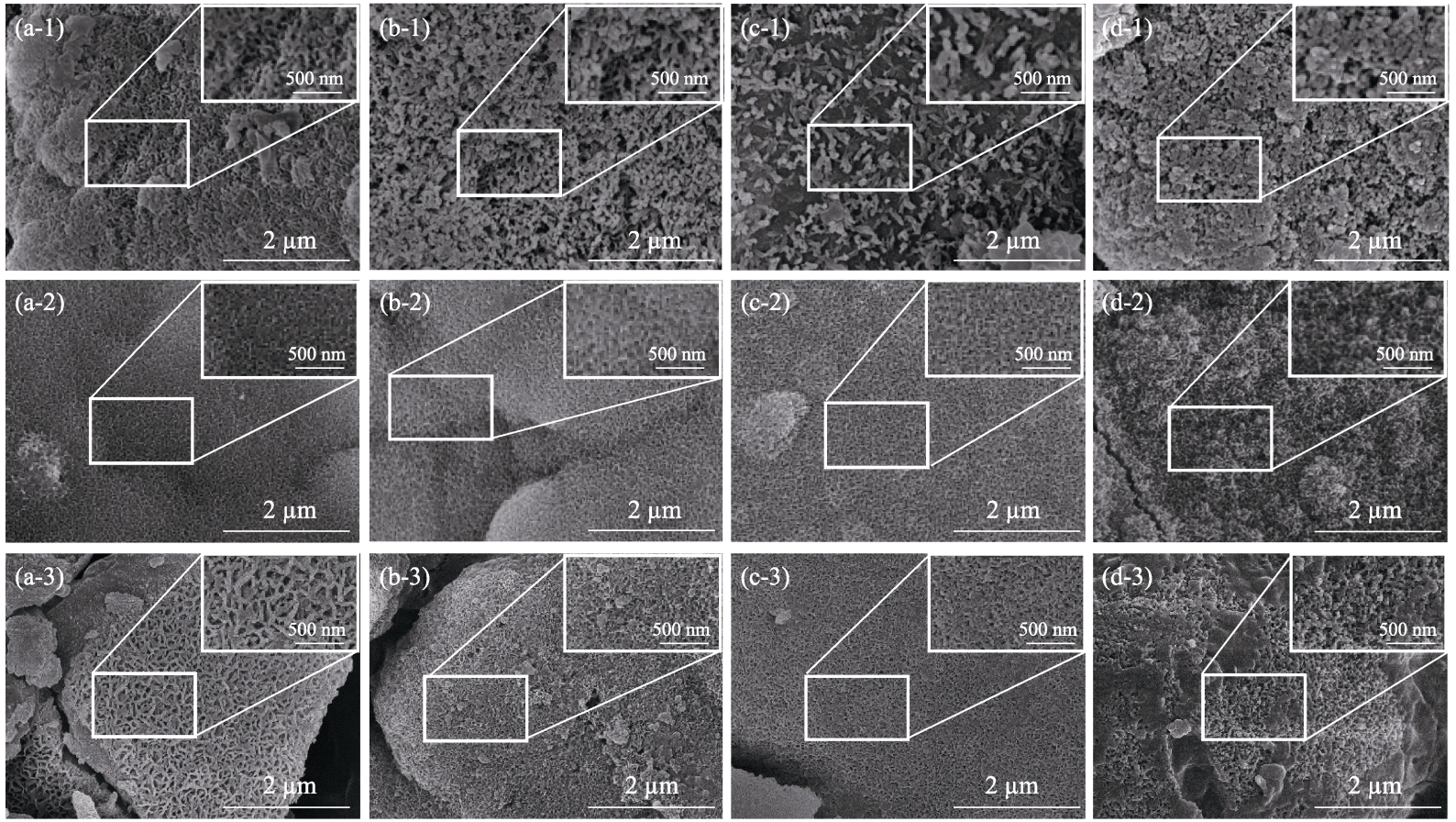

图 2 (a)天冬氨酸、(b)甘氨酸、(c)赖氨酸浓度为 $0.1 \mathrm{~mol} / \mathrm{L}$ 时和(d)对照组骨水泥矿化(1) $5 、$ (2) 7 和(3) $14 \mathrm{~d}$ 后表面形貌

Fig. 2 Surface morphologies of BBG bone cements after being soaked in 1.5SBF containing $0.1 \mathrm{~mol} / \mathrm{L}$ (a) aspartic acid, (b) glycine, (c) lysine, and (d) control group without amino acid for (1) 5, (2) 7 and (3) $14 \mathrm{~d}$

较小的棒状矿物组成的网状结构, 对照组是由偏球 形的颗粒形成的网状结构。实验结果与文献中记载 的酸性、中性和碱性氨基酸在低浓度下对 HA 晶体 生长过程中形貌的调控结果基本一致 ${ }^{[4]}$, 在含有酸 性氨基酸天冬氨酸和碱性氨基酸赖氨酸的调控下形 成了条状的晶体。从浸泡 $5 \mathrm{~d}$ 后产物的 XRD 图谱 (图 3(a))中可以看到, 天冬氨酸组产物已经出现了 $\mathrm{HA}$ 的特征峰, 但结晶度较低, 其他组产物均为馒头 峰。浸泡 $14 \mathrm{~d}$ 后(图 3(b)), 四组骨水泥浸泡产物均 显示出 HA 的特征峰, 而天冬氨酸组产物的结晶度 明显高于其他组的产物。据文献[19-20]报道, 酸性 氨基酸在 HA 的合成中具有较为突出的调控效应, 可以推测上述现象是因为相对于其他氨基酸, 天冬氨 酸在使得 HA 特定晶面特异性生长过程中的作用更
明显，导致特定晶面的衍射峰强度增大。

氨基酸对 HA 矿化产物形貌的影响与它们在溶 液中的等电点有关 ${ }^{[21]}$ 。如图 4(a)所示, 随着 BBG 在 $\mathrm{SBF}$ 溶液中降解, 浸泡 $2 \mathrm{~d}$ 后含有天冬氨酸的微环 境 $\mathrm{pH}$ 升高至 7.94 , 而天冬氨酸的等电点为 2.77 , 因 此在碱性环境中天冬氨酸被电离出 $\mathrm{H}^{+}$并带有负电。 由于 $\mathrm{HA}$ 晶体的生长基元属于离子配位体结构, $c$ 轴 的生长基元主要是带负电荷的 $\mathrm{Ca}-\mathrm{P}_{6} \mathrm{O}_{24}, a$ 轴和 $b$ 轴 的生长基元主要是带正电荷的 $\mathrm{OH}-\mathrm{Ca}_{6}{ }^{[22]}$ 。因此，当 天冬氨酸吸附在 HA 晶体的 $c$ 轴上时, $c$ 轴方向晶体 的生长就会受到抑制。但由于 $a 、 b$ 轴方向生长基元 尺寸较小 ${ }^{[23]}$, 在前期尽管存在抑制 $c$ 轴生长的因素, 最终仍是 $c$ 轴方向生长速率较快, 因此, 前期矿化 产物的形貌呈长条形。然而, 随着浸泡时间的 

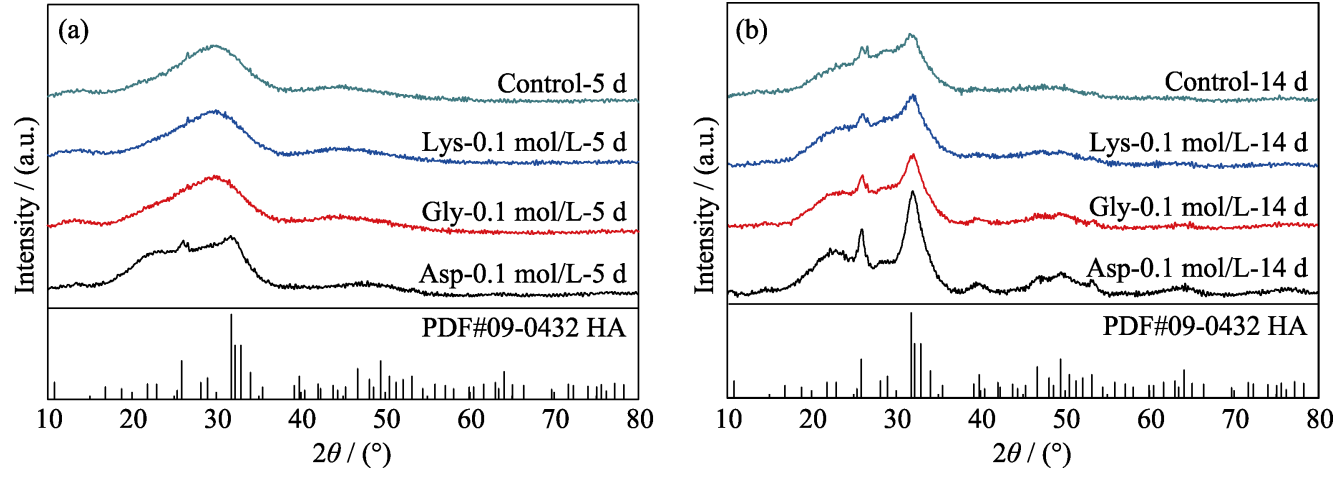

图 3 骨水泥在含有 $0.1 \mathrm{~mol} / \mathrm{L}$ 氨基酸的 $1.5 \mathrm{SBF}$ 中浸泡(a) 5 和(b) $14 \mathrm{~d}$ 后产物的 XRD 图谱

Fig. 3 XRD patterns of the products produced by soaking bone cements in $1.5 S B F$ containing $0.1 \mathrm{~mol} / \mathrm{L}$ amino acid for (a) 5 and (b) $14 \mathrm{~d}$
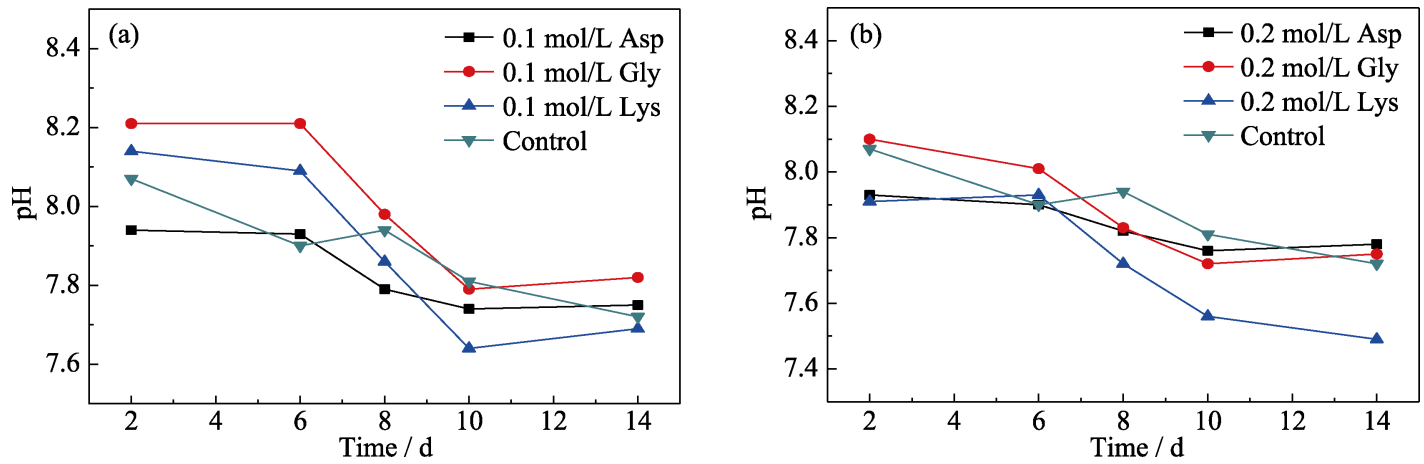

图 4 骨水泥在含有(a) 0.1 和(b) $0.2 \mathrm{~mol} / \mathrm{L}$ 氨基酸的 $1.5 \mathrm{SBF}$ 中矿化过程的 $\mathrm{pH}$ 变化

Fig. $4 \mathrm{pH}$ Changes of 1.5SBF containing (a) 0.1 and (b) $0.2 \mathrm{~mol} / \mathrm{L}$ amino acid during the mineralization of bone cements

延长, $a 、 b$ 轴方向的晶体生长逐渐超过 $c$ 轴方向, 最 终形成由条状晶体组成的片状结构。甘氨酸的等电 点为 5.97 , 与天冬氨酸在浸泡溶液中同样带负电荷, 但对 $\mathrm{HA}$ 晶体生长的影响较小。赖氨酸等电点为 9.74, 浸泡 $2 \mathrm{~d}$ 溶液的 $\mathrm{pH}$ 由 7.40 升高至 8.09 , 这一 过程中赖氨酸带正电荷, 从图 4(a)中可以看到, 随 着浸泡时间的延长，溶液 $\mathrm{pH}$ 降低至 7.7 左右，与其 他组相比更低，而赖氨酸更容易结合 $\mathrm{OH}^{-}$，从而降 低溶液 $\mathrm{pH}, \mathrm{HA}$ 的 $a 、 b$ 轴方向的生长基元 $\mathrm{OH}-\mathrm{Ca}_{6}$ 减少，使得晶体沿 $c$ 轴的生长速率较快，形成米粒 状或棒状。

\section{2 氨基酸浓度对浸泡物 BBG 骨水泥表面形 貌的影响}

将 BBG 骨水泥样品分别浸泡在含 $0.2 \mathrm{~mol} / \mathrm{L}$ 的 天冬氨酸、甘氨酸和赖氨酸的模拟体液中一定时间 后, 表征分析其矿化产物的形貌和物相(图 5)。浸泡 $5 \mathrm{~d}$ 后, 含有氨基酸的骨水泥表面几乎没有形成沉 积; 浸泡 $7 \mathrm{~d}$ 后, 天冬氨酸组和赖氨酸组样品表面出 现了颗粒状的小团块体, 而甘氨酸组样品表面形成 了小颗粒组成的疏松网状结构; 浸泡 $14 \mathrm{~d}$ 后，甘氨 酸组样品表面的矿化产物增多, 形成较为完整的网 状结构, 而天冬氨酸组和赖氨酸组样品表面则形成
了偏向于小球形的矿化产物。

与 $0.1 \mathrm{~mol} / \mathrm{L}$ 氨基酸浓度相比, 当氨基酸浓度增 大时, 矿化产物的形貌发生了明显的变化。氨基酸 浓度提高后, 与溶液中 $\mathrm{Ca}^{2+} 、 \mathrm{Mg}^{2+}$ 离子的结合增多, 对 HA 各晶面的抑制作用也有所增强，导致骨水泥 浸泡 $5 \mathrm{~d}$ 后表面几乎无法形成矿物沉积。天冬氨酸 和赖氨酸浓度提高后, 矿物的长径比明显减小。其 中天冬氨酸组浸泡 $14 \mathrm{~d}$ 后产物呈球形, 与文献[23] 报道的在较高天冬氨酸浓度下 $\mathrm{HA}$ 的生长结果相一 致, 这是由于天冬氨酸浓度的增大, 对 $\mathrm{HA}$ 晶体 $c$ 轴 方向的生长产生了更强的抑制作用，降低了晶体沿 $c$ 轴方向的生长速度。但是由于 $\mathrm{OH}-\mathrm{Ca}_{6}$ 比 $\mathrm{Ca}-\mathrm{P}_{6} \mathrm{O}_{24}$ 的尺寸小, 导致晶体沿 $a 、 b$ 和 $c$ 轴方向生长速度 趋于一致, 使得最终的矿化产物呈球形。对于甘氨 酸组, 由于甘氨酸浓度增加对晶体 $\mathrm{c}$ 轴方向上生长 的抑制作用增强，导致晶体的尺寸较小。对于赖氨 酸组, 从图 4(b)中可以看到, 浸泡 $10 \mathrm{~d}$ 后, 溶液 $\mathrm{pH}$ 降低至 7.5 以下, 相比 $0.1 \mathrm{~mol} / \mathrm{L}$ 氨基酸浓度时更低, 可能是由于氨基酸浓度增加导致带正电荷的赖氨 酸吸附了溶液中更多的 $\mathrm{OH}^{-}$, 进一步降低了溶液 的 $\mathrm{pH}$ 。

从骨水泥在不同浓度氨基酸溶液中矿化产物的 XRD 图谱(图 6)对比来看, 在两种不同浓度氨基酸 

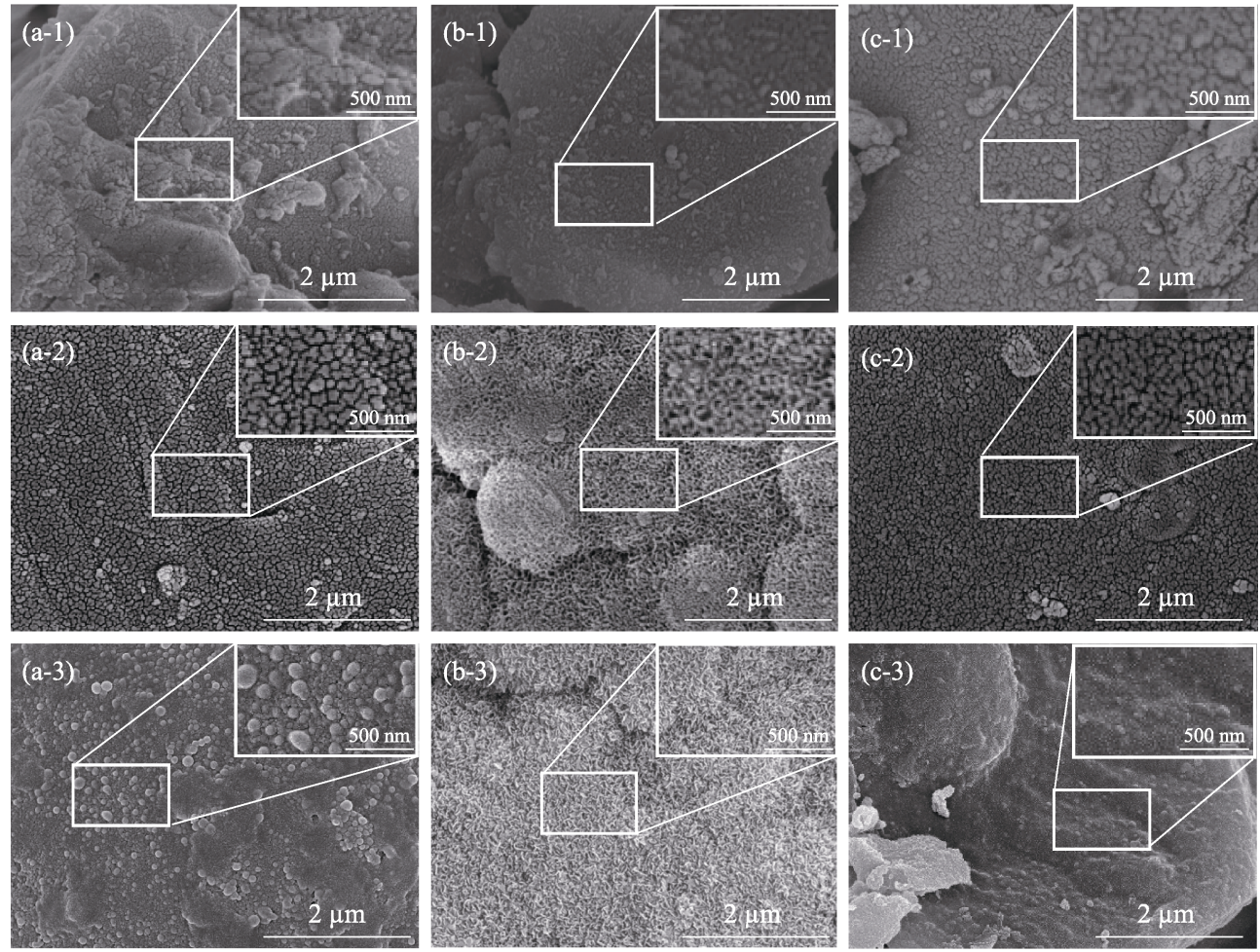

图 5 (a)天冬氨酸、(b)甘氨酸、(c)赖氨酸浓度为 $0.2 \mathrm{~mol} / \mathrm{L}$ 时骨水泥矿化(1) 5、(2) 7 和(3) $14 \mathrm{~d}$ 后表面形貌

Fig. 5 Surface morphologies of BBG bone cements after being soaked in 1.5SBF containing $0.2 \mathrm{~mol} / \mathrm{L}$

(a) aspartic acid, (b) glycine and (c) lysine for (1) 5, (2)7 and (3) $14 \mathrm{~d}$
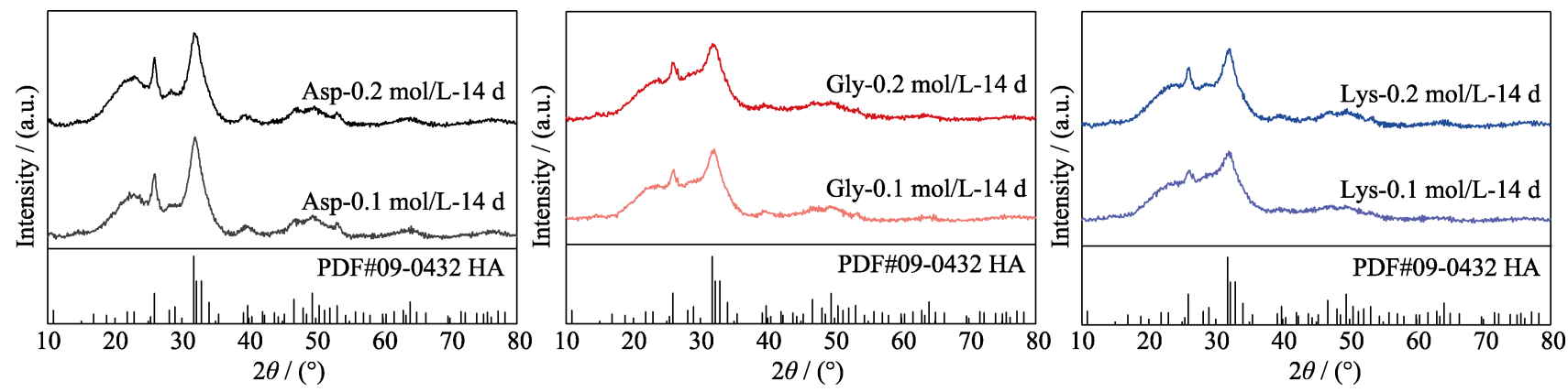

图 6 骨水泥在不同浓度氨基酸溶液中矿化产物 XRD 图谱

Fig. 6 XRD patterns of the products produced in 1.5SBF with different amino acids at different concentrations

的 SBF 溶液中浸泡 $14 \mathrm{~d}$ 所形成的矿化产物的 XRD 图谱强度相近。在 $2 \theta$ 为 $31^{\circ}$ 和 $26^{\circ}$ 附近分别出现 HA 晶体的最强峰及次强峰, 对应晶体的(211)和 (002)晶面。根据文献[6]报道，通过计算晶体中不同 衍射峰的相对强度可以分析晶体生长的取向度。为 分析氨基酸对矿化产物生长晶面的特异性吸附, 本 研究对这两个峰的相对强度作了比较(图 7)。其中在 $0.2 \mathrm{~mol} / \mathrm{L}$ 天冬氨酸溶液矿化产物衍射峰的相对强 度较 $0.1 \mathrm{~mol} / \mathrm{L}$ 的相对强度更低, 表明 $c$ 轴方向的晶 体生长受到了更强的抑制, 造成了图 5 所示的形貌。 $0.1 \mathrm{~mol} / \mathrm{L}$ 氨基酸组矿化产物两个峰的相对强度较 高, $0.2 \mathrm{~mol} / \mathrm{L}$ 氨基酸组次之, 空白对照组最低。在各 浓度氨基酸组中, 天冬氨酸矿化产物两个峰的相

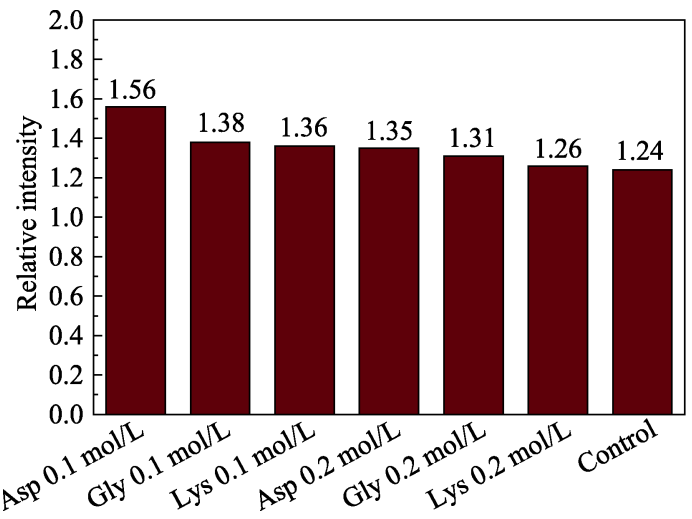

图 7 骨水泥在不同浓度氨基酸溶液中矿化产物(211)和(002) 晶面的 XRD 峰相对强度

Fig. 7 Relative intensities of (211) and (002) peaks in XRD patterns of mineralization products 
对强度较高, 甘氨酸次之, 赖氨酸最低。这一结果同 样验证了氨基酸是通过在晶体表面特异性吸附, 从 而影响晶体在各晶面方向上的生长。

\subsection{BBG 在酸性 2Mg-1.5SBF 溶液中的复相} 矿化

将硼硅酸盐生物玻璃粉末压片后，动态浸泡在 $70{ }^{\circ} \mathrm{C} 、 \mathrm{pH} 3.5$ 的 $2 \mathrm{Mg}-1.5 \mathrm{SBF}$ 溶液中 7 和 $10 \mathrm{~d}$ 后, 对 矿化产物进行表征分析。从图 8 可以观察到, 动态 浸泡 $7 \mathrm{~d}$ 的矿化产物中既有 HA 的特征峰(PDF\#090432), 也有白磷钙矿的特征峰(PDF\#70-2064), 说 明浸泡物表面产物中同时包含了 $\mathrm{HA}$ 和 WH 矿物。 在浸泡 $10 \mathrm{~d}$ 样品的 XRD 图谱中, $\mathrm{HA}$ 和 $\mathrm{WH}$ 晶体的 衍射峰强度进一步增强。作为第二丰富的骨矿物, WH 具有不同于 HA 的晶体结构。WH 晶体属于三方 晶系, 晶格参数为 $a=b=1.0350 \mathrm{~nm}$ 和 $c=3.7085 \mathrm{~nm}$; 而 $\mathrm{HA}$ 晶体为六方晶系, 晶格参数为 $a=b=0.9418 \mathrm{~nm}$ 和 $c=0.6884 \mathrm{~nm}^{[24]}$ 。根据浸泡 $7 \mathrm{~d}$ 的嗍硅酸盐生物玻 璃压片的 TEM 选区电子衍射花样(图 9), 两种晶体 的晶面分别是(100)、(01 $\overline{1}) 、(11 \overline{1})$, 和 $(12 \overline{1})$ 、 $(2 \overline{1} 0) 、(31 \overline{1})$, 与 HA 和 WH 的 PDF 卡片相对应, 再 次验证了产物中存在 HA 和 WH 两种晶体。

图 10 是 BBG 压片在 $2 \mathrm{Mg}-1.5 \mathrm{SBF}$ 溶液中浸泡 $7 \mathrm{~d}$ 后的 SEM 照片。由图可见, 浸泡物表面形成片 状团簇, 这和 HA 晶体的形貌相符合。但无法直接观 察到白磷钙矿晶体的形貌, 可能是由于生长的 $\mathrm{WH}$ 晶体的晶粒尺寸较小，且被周围生长情况较好的 $\mathrm{HA}$ 晶体包覆, 因此难以直接辨认。WH 在生物体的 硬组织中以短程而有序的状态存在，难以将纯 WH 从硬组织中分离出来 ${ }^{[25]}$, 但可以通过 XRD、TEM 选 区电子衍射花样等方法进行检测分析。

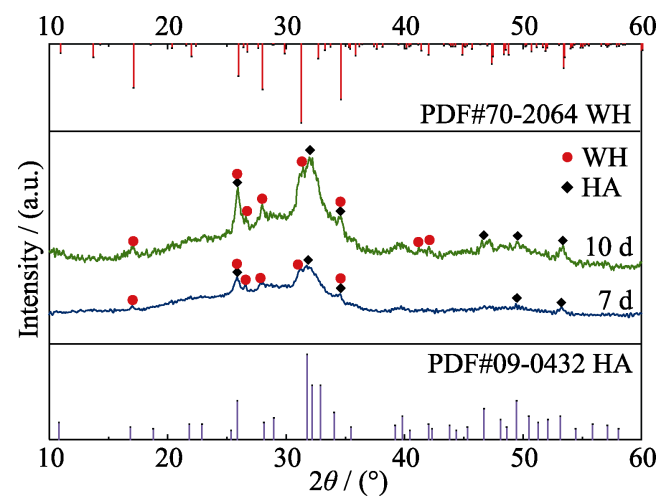

图 8 BBG 动态浸泡 7 和 $10 \mathrm{~d}$ 后产物的 XRD 图谱以及 $\mathrm{HA}$ 和 WH 的标准 XRD 图谱

Fig. 8 XRD patterns of BBG after dynamic soaking for 7 and $10 \mathrm{~d}$ against the standard XRD patterns of $\mathrm{HA}$ and $\mathrm{WH}$
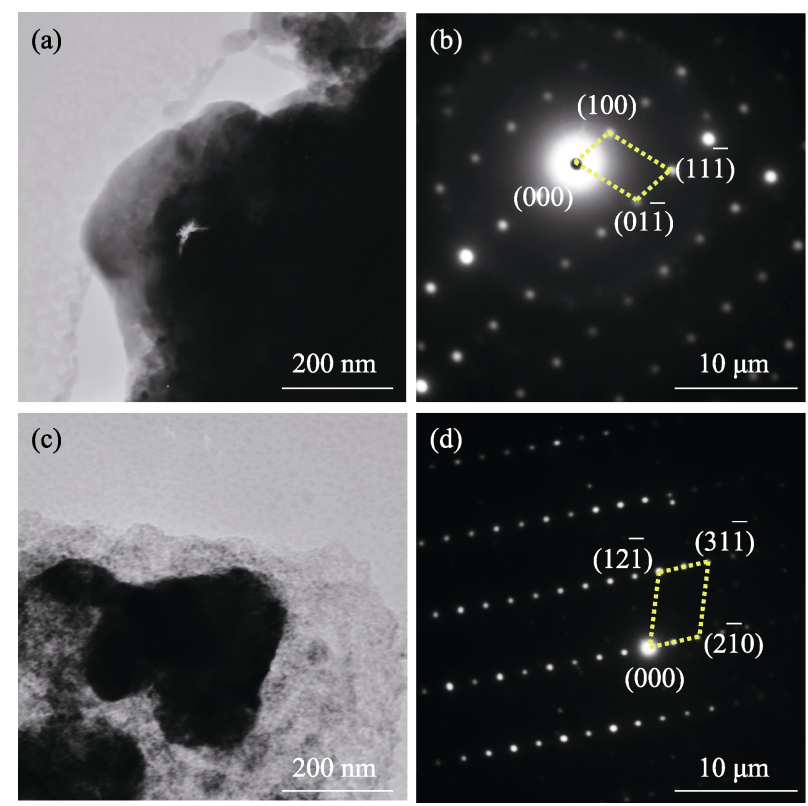

图 9 矿化产物中(a, b) HA 和(c, d) WH 的(a, c) TEM 照片和 (b, d)选区电子衍射花样

Fig. 9 (a, c) TEM images and (b, d) selected area electron diffraction patterns of mineralized (a, b) HA and (c, d) WH

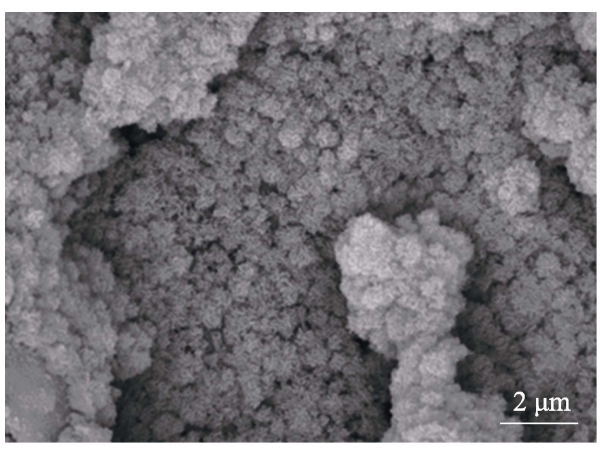

图 10 BBG 动态浸泡在 2Mg-1.5SBF 矿化 $7 \mathrm{~d}$ 后产物表面 SEM 形貌照片

Fig. 10 SEM image of BBG mineralized surface after being immersed in 2Mg-1.5SBF for $7 \mathrm{~d}$

在浸泡初期，随着 $\mathrm{BBG}$ 的快速降解，溶液 $\mathrm{pH}$ 开 始呈现上升趋势，但因使用本实验室自主设计的动 态浸泡系统，浸泡环境在较短时间后又回归偏酸性， 满足矿化形成 WH 的酸碱度条件 ${ }^{[16]}$ 。由于溶液中含 有较多的 $\mathrm{Mg}^{2+}$, 同时 BBG 降解释放的 $\mathrm{Mg}^{2+}$ 也会进 入溶液中, 确保有足够的 $\mathrm{Mg}^{2+}$ 离子达到矿化形成 WH 所需的过饱和度。此外, 溶液的温度设定也辅助 WH 形成。当然 WH 生成并未完全影响因生物玻璃 降解而导致骨水泥附近区域的 $\mathrm{pH}$ 升高, 这也确保了 浸泡体表面 HA 晶体的形成。随着浸泡时间的延长, 玻璃持续降解释放离子，两种晶体都在不断地形核、 生长, 并最终形成具有自然骨组织所特有的 HA 和 WH 的复相矿化组成。有研究表明, 当复相矿物中的 
HA 和 WH 以 $3: 1$ 的比例存在时, 对细胞增殖和成骨 分化有明显的促进作用 ${ }^{[26]}$ 。

\section{3 结论}

将硼硅酸盐生物玻璃基骨水泥浸泡在不同的 SBF 溶液中会产生不同的矿化过程, 尤其是加入不 同的氨基酸或改变氨基酸的浓度, 对矿化过程及矿 物的形貌都会有一定的影响。在 HA 晶体生长过程 中, 天冬氨酸在(001)晶面上有较强的吸附作用, 浓 度增大后 $c$ 轴方向晶体生长的速度减缓, 晶体的长 径比减小; 甘氨酸导致骨水泥表面形成的晶体尺寸 较小; 赖氨酸容易与 $\mathrm{OH}^{-}$结合, 导致 $\mathrm{HA}$ 晶体的 $a$ 、 $b$ 轴方向的生长基元 $\mathrm{OH}-\mathrm{Ca}_{6}$ 减少, 骨水泥表面最终 形成米粒状或棒状的 HA 晶体。氨基酸浓度的改变 主要使得氨基酸在 HA 不同晶面上吸附作用强度发 生变化, 从而影响矿化产物的形貌。此外, 通过调整 $\mathrm{SBF}$ 溶液的成分、 $\mathrm{pH}$ 和环境温度, 在动态浸泡实验 中可以获得类似于自然骨组织中的 HA/WH 复相矿 物。这对于进一步精细调控 SBF 的成分、酸碱度及 温度来体外模拟自然环境下 BBG 基骨水泥的矿化 行为, 从而获得具有更好成骨性能的玻璃基骨水泥 具有重要意义。

\section{参考文献:}

[1] KOKUBO T, TAKADAMA H. How useful is SBF in predicting in vivo bone bioactivity? Biomaterials, 2006, 27(15): 2907-2915.

[2] YILMAZ B, PAZARCEVIREN A E, TEZCANER A, et al. Historical development of simulated body fluids used in biomedical applications: A review. Microchemical Journal, 2020, 155: 104713.

[3] PALAZZO B, WALSH D, IAFISCO $\mathrm{M}$, et al. Amino acid synergetic effect on structure, morphology and surface properties of biomimetic apatite nanocrystals. Acta Biomaterialia, 2009, 5(4): 1241-1252.

[4] HUANG S, ZHOU K, LI Z. Inhibition mechanism of aspartic acid on crystal growth of hydroxyapatite. Transactions of Nonferrous Metals Society of China, 2007, 17(3): 612-616.

[5] MARTINS M L, IESSI I L, QUINTINO M P, et al. Glucose is an active chemical agent on degradation of hydroxyapatite nanostructure. Materials Chemistry and Physics, 2020, 240: 122166.

[6] LI Z C, REN Q, CUI J Y, et al. Comparing the efficacy of hydroxyapatite nucleation regulated by amino acids, poly-amino acids and an amelogenin-derived peptide. CrystEngComm, 2020, 22: 3814-3823.

[7] WU Y Y, YE S, YAO A H, et al. Effect of gas-foaming porogen$\mathrm{NaHCO}_{3}$ and citric acid on the properties of injectable macroporous borate bioactive glass cement. Journal of Inorganic Materials, 2017, 32(7): 777-784.

[8] XIE X, PANG L B, YAO A H, et al. Nanocement produced from borosilicate bioactive glass nanoparticles composited with alginate. Australian Journal of Chemistry, 2019, 72(5): 354-361.
[9] CUI X, ZHAO C J, GU Y F, et al. A novel injectable borate bioactive glass cement for local delivery of vancomycin to cure osteomyelitis and regenerate bone. Journal of Materials Science-Materials in Medicine, 2014, 25(3): 733-745.

[10] JANG H L, JIN K, LEE J, et al. Revisiting whitlockite, the second most abundant biomineral in bone: nanocrystal synthesis in physiologically relevant conditions and biocompatibility evaluation. ACS Nano, 2014, 8(1): 634-641.

[11] LUNA-DOMINGUEZ J H, TELLEZ-JIMENEZ H, HERNANDEZCOCOLETZI $\mathrm{H}$, et al. Development and in vivo response of hydroxyapatite/whitlockite from chicken bones as bone substitute using a chitosan membrane for guided bone regeneration. Ceramics International, 2018, 44(18): 22583-22591.

[12] KIM H D, JANG H L, AHN H Y, et al. Biomimetic whitlockite inorganic nanoparticles-mediated in situ remodeling and rapid bone regeneration. Biomaterials, 2017, 112: 31-43.

[13] CHANG Y, ZHAO R, WANG $\mathrm{H}$, et al. A novel injectable whitlockite-containing borosilicate bioactive glass cement for bone repair. Journal of Non-Crystalline Solids, 2020, 547: 120291-1-11.

[14] YAO A H, WANG D P, HUANG W H, et al. In vitro bioactive characteristics of borate-based glasses with controllable degradation behavior. Journal of the American Ceramic Society, 2007, 90(1): 303-306.

[15] MARQUES M R C, LOEBENBERG R, ALMUKAINZI M. Simulated biological fluids with possible application in dissolution testing. Dissolution Technologies, 2011, 18(3): 15-28.

[16] ZHANG J T, LIU W Z, SCHNITZLER V, et al. Calcium phosphate cements for bone substitution: chemistry, handling and mechanical properties. Acta Biomaterialia, 2014, 10(3): 1035-1049.

[17] ANDERSEN T, STRAND B L, FORMO K, et al. Alginates as biomaterials in tissue engineering. Carbohydrate Chemistry, 2011, 37: 227-258.

[18] JANG H L, LEE H K, JIN K, et al. Phase transformation from hydroxyapatite to the secondary bone mineral, whitlockite. Journal of Materials Chemistry B, 2015, 3(7): 1342-1349.

[19] IKAWA N, KIMURA T, OUMI Y, et al. Amino acid containing amorphous calcium phosphates and the rapid transformation into apatite. Journal of Materials Chemistry, 2009, 19(28): 4906-4913.

[20] ZHANG G D, CHEN J D, YANG S, et al. Preparation of aminoacid-regulated hydroxyapatite particles by hydrothermal method. Materials Letters, 2011, 65(3): 572-574.

[21] 周杨. 羟基磷灰石微纳结构的制备及应用研究. 上海: 中国科 学院上海硅酸盐研究所博士学位论文, 2018.

[22] GUO Y, LI Y, LI L. Mechanism of nano hydroxyapatite crystallization under hydrothermal conditions. Journal of Civil Aviation University of China, 2007, 25(4): 26-30.

[23] WANG Y, WU J, RUAN Y, et al. Influence of aspartic acid concentration on shape of HAP. Technology and Development of Chemical Industry, 2017, 46(8): 21-23, 53.

[24] WANG C F, JEONG K J, PARK H J, et al. Synthesis and formation mechanism of bone mineral, whitlockite nanocrystals in tri-solvent system. Journal of Colloid and Interface Science, 2020, 569: 1-11.

[25] JANG H L, BIN ZHENG G, PARK J, et al. In vitro and in vivo evaluation of whitlockite biocompatibility: comparative study with hydroxyapatite and beta-tricalcium phosphate. Advanced Healthcare Materials, 2016, 5(1): 128-136.

[26] CHENG H, CHABOK R, GUAN X F, et al. Synergistic interplay between the two major bone minerals, hydroxyapatite and whitlockite nanoparticles, for osteogenic differentiation of mesenchymal stem cells. Acta Biomaterialia, 2018, 69: 342-351. 